\title{
Better medicine or more medicine, where are we heading?
}

Undoubtedly, at present medicine is going through an era where at least two opposing pathways "fight" for predominance. In this situation, and as has always done, the medical profession plays an essential role when deciding on the most appropriate approach for patients, above any interest whatsoever, or on the other hand, giving priority to other interests. Many changes have taken place in the practice of medicine for decades, changes that have become more remarkable after World War II; and several have been very beneficial for people's health, mostly due to the significant scientific advances in disease prevention, diagnosis and treatment. However, they have been affected by multiple external factors and, in addition to the benefits obtained,other effects emerged, which were not very promising for the population nor for the medical profession.

I would like to provide some insight regarding one of the latter aspects, insight that is related to the pathways I mentioned at the beginning. In my opinion, there are two opposing pathways, one is taking us to a better medicine and the other one is leading us to having more medicine.

\section{What do we mean by better medicine?}

I believe that this should not require further explanations, although I am not sure this is always the case. Good medicine is that which people really need and is mainly based on the following principles: that health maintenance is its main objective, that the humanistic vision where comprehension, aid and patients' care prevail during the provision of healthcare, that professionals have the necessary knowledge, and that the original and essential ethical principles of the medical profession are kept. It is easier to define it than to comply with these rules; therefore, we have to strongly struggle so that the ancient drive of medicine does not wane, "the care of health and the relief of those who suffer," or otherwise we would be gradually moving away from the principle of better medicine.

\section{What do we mean by more medicine?}

This definition is made up of several components, but I will only point out the ones I consider as the most relevant today. One of the important aspects is the growing medicalization in people's life, a subject that I have already mentioned in a recently published editorial (Arch Argent Pediatr, December 2012 ) and therefore I am not going to refer to the overall concepts but just focus on the most pernicious ones. The main aspect of this medicalization is the non-scientifically supported overdiagnosis of certain conditions, especially through some early screenings of "likely" future diseases. There are multiple pieces of evidence that medicine has turned detrimental to healthy people through earlier and earlier routine screenings. Likewise, overdiagnosis results in the overprescription of unnecessary medications with the risk of leading to overdose related adverse events. Another critical aspect to be pointed out is the constant broadening of disease definition, which might encourage behaviors that lead to "inventing" diseases, which undoubtedly will be more damaging than beneficial.

Some screening programs are detecting cancers at an early stage that neither cause symptoms nor will lead to mortality. Through very sensitive diagnostic technologies, very small "abnormalities" are detected, but they are so tiny that they will always remain benign. Likewise, the broadening of disease definition makes "new" diseases appear, with no sound support at all, and many times they make people who are not really sick receive long-term, and sometimes even continuous, treatment. As pediatricians, we have the example of the unnecessary use of drugs in children with Attention Deficit Hyperactivity Disorder (ADHD), which many investigators consider erroneous and excessive given that most of the times this is a temporary disorder that requires no treatment, but support and advice to families, or non pharmacological treatments.

The gradual increase in scientific publications clearly demonstrates the growing number of individuals who are harmed by overdiagnosis and excessive, and many times, unnecessary treatments, as well as by the medicines being administered, which could be avoided most of the times and that are commonly given in excessive doses that result in adverse events. Physicians are frequently prone to prescribing new medicines believing that these are better than the ones used in the past, which is a misleading fact. It has been shown that today new medicines producing a remarkable and beneficial change to the patient are truly exceptional, even though they are all 
portrayed as wonderful at the time of their release. Additionally, such excessive and unnecessary drug prescription makes doubtful the probity of some professionals who prioritize their personal interests and harmonize them with the MedicalIndustrial Complex interests, so much in fashion today. In 2012 a USA organization published an extensive list of physicians who received fees from the pharmaceutical industry; several of them had been paid sums well over USD 500,000 per year.

In an excellent editorial on the overdiagnosis of diseases that are not such (BMJ, May 2012) authors underscore, among several insights, the fact that "more honest information is needed about the risk of overdiagnosis, particularly in relation to screening; more deeply, mounting evidence that we're harming healthy people may force a questioning of our faith in ever-earlier detection, a renewal of the process of disease definition, and a fundamental shift in the systemic incentives driving dangerous excess."

This aspect is accompanied by the excessive use of unnecessary tests, sometimes by ignorance, some others by convenience (very brief visits, little dialogue with the patient) and yet others by a financial interest of the professional. Recent data from USA point out that approximately $80 \%$ of the health care expenditures depends on physicians' decisions and that overprescription in unnecessary tests and procedures is one of the main causes of the high cost of healthcare in the USA, which is much higher than in other countries with a better and more equal health system. Besides, in the context of such commodified care, similar in many aspects to that of Argentina, where the market seems to reign over other aspects, it is not strange that the prescription of unnecessary tests and procedures has become an excellent business.
Undoubtedly when organizing medicine as a business, one of the greatest interferences occurs in the doctor-patient relationship, that we all know it surely is the mainstay which good medicine is based upon.

I do not want to speak at length about this extensive subject which is part of the ethical principles of our profession, so much beaten today. I will only quote an outstanding statement by Julian Tudor Hart, prestigious British physician who practiced general medicine many years. In 1971 he published in the Lancet a paper called The Inverse Care Law, from which I have picked the following quotation: "The availability of good medical care tends to vary inversely with the need for it in the population. This inverse care law operates more completely where medical care is most exposed to market forces, and less so where such exposure is reduced."

It is necessary to understand that us, physicians, are the ones who should strive to change this reality in each of the deeds we do because it clearly distorts the goals of medicine. In a time when apparent "miracles" in medical care are dazzling us and we are overawed by the overwhelming technology, and also when the ethical principles of our profession are staggering, the silent and humble work of most of the physicians can change the course of this growing and pernicious situation that today is overwhelming all of us who wish for a better medicine.

José M. Ceriani Cernadas Editor

http:/ /dx.doi.org/10.5546/aap.2013.370 